\title{
Verbal learning and memory deficits in Mild Cognitive Impairment
}

\author{
F. Ribeiro, ${ }^{1,2}$ M. Guerreiro, ${ }^{1,2}$ and A. De Mendonça ${ }^{1,3}$ \\ ${ }^{1}$ Dementia Clinics, Institute of Molecular Medicine and Faculty of Medicine of Lisbon, Lisbon, \\ Portugal \\ ${ }^{2}$ Laboratory of Language, Institute of Molecular Medicine and Faculty of Medicine of Lisbon, \\ Lisbon, Portugal \\ ${ }^{3}$ Neurology Department and Laboratory of Neurosciences, Institute of Molecular Medicine and \\ Faculty of Medicine of Lisbon, Lisbon, Portugal
}

\begin{abstract}
Criteria for amnestic MCI rely on the use of delayed recall tasks to establish the presence of memory impairment. This study applied the California Verbal Learning Test to detail memory performance in MCI patients $(n=70)$, as compared to control subjects $(n=92)$ and AD patients $(n=21)$. Learning across the 5 trials was different among the 3 groups. Learning strategy was also different, the MCI group showing less semantic clustering than the control group. However, both MCI patients and controls could benefit from semantic cueing. This study showed that beyond consolidation deficits, MCI patients have marked difficulties in acquisition and recall strategies.
\end{abstract}

\section{INTRODUCTION}

Memory complaints are common in aged people. Some of these aged people experience changes severe enough to bring them to the medical doctor, who nowadays commonly establishes the diagnosis of Mild Cognitive Impairment (MCI) (de Mendonça, Guerreiro, Ribeiro, Mendes, \& Garcia, 2004). MCI patients typically present with memory complaints of unknown etiology and perform below the norms for age and education on neuropsychological memory tests. Nevertheless they have a relatively normal general cognitive function, maintain their activities of daily living and are not demented (Petersen et al., 1999). Mild Cognitive Impairment, particularly the amnestic type (Petersen et al., 2001a), appears to represent a transition between normal aging and early dementia, since in clinical settings patients with MCI show rates of conversion to dementia, usually Alzheimer's disease (AD), of about $12 \%$ per year (Petersen et al., 1999). Criteria for amnestic MCI make use of scores in delayed recall of episodic memory tasks to establish the presence of memory impairment (Win- blad et al., 2004; see Arnaiz \& Almkvist, 2003 for a review on memory deficits in MCI and AD). However, poor delayed recall can reflect deficits in distinct memory processes: encoding, consolidation and retrieval. The contribution of these processes to the observed deficit is still unclear (Arnaiz \& Almkvist, 2003). Deficits in semantic memory were also found in $\mathrm{MCI}$ as in AD patients (Dudas, Claghe, Thompson, Graham, \& Hodges, 2005). Furthermore, recent work has called attention to the relevance of examining memory deficits in areas other than declarative memory, by using verbal and nonverbal priming tests (Perri et al., 2005). Impairments in other cognitive domains, such as orientation, attention, executive functions and visuospatial abilities, may also be found in patients with MCI (Grundman et al., 2004; Ribeiro, Guerreiro, \& de Mendonça, in press). Detailed knowledge of the memory processes disturbed in MCI should contribute to the understanding of the pathophysiology of MCI, and help to delineate future rehabilitation interventions in these patients.

In order to characterize the MCI memory profile under the present study, a well known test of verbal

Address correspondence to Filipa Ribeiro, Laboratory of Language, Faculty of Medicine of Lisbon, Av. Prof. Egas Moniz, 1649-028 Lisbon, Portugal (E-mail: piparibeiro@mail.telepac.pt). 
learning and memory, the California Verbal Learning Test (CVLT; Delis, Kramer, Kaplan, \& Ober, 1987) was chosen. The CVLT was developed to assist in the diagnosis of memory disorders. Numerous studies have found the CVLT helpful for detecting and characterizing the memory deficits related with many neurological disorders, including Alzheimer's disease and other dementing disorders (Delis et al. 1991; Kramer, Levin, Brandt, \& Delis, 1989). The test does more than evaluating learning and memory impairments; it was also designed to assess the mechanisms responsible for those difficulties (Lezak, 1995). The CVLT is a five trial shopping list learning test with immediate and delayed recall, both free and semantically cued. The list consists of four items from each of four semantically distinct categories (fruits, herbs and spices, clothing and tools) arranged in a way such that adjacent words on the list are from different categories. The semantic structure of the CVLT list allows for the assessment of learning strategies that would not be possible with lists of unrelated words. Effective learning is related to the capacity to use that semantic structure by actively clustering words of the same semantic group (semantic clustering). The CVLT also includes a cued recall task after the initial free recall and before the delayed recall. In this cued recall task the experimenter makes the semantic structure explicit when he asks the subject to recall items from each of the four list categories. This procedure should facilitate both recall and subsequent clustering. The CVLT structure is well suited not only to study consolidation deficits but also to test acquisition difficulties. Considering the data on executive and semantic deficits in MCI, we hypothesized that MCI patients might display an acquisition deficit related to weak implementation of semantic strategies in learning.

\section{METHODS}

Participants were patients with Mild Cognitive Impairment and patients with $\mathrm{AD}$ attending the Dementia Outpatient Clinic, Hospital Santa Maria, and a Memory Clinic, both in Lisbon. Control subjects were elderly people, who worked as volunteers in the hospital or attended two senior universities in Lisbon area. The study was approved by the local ethics committee.

\section{$\mathrm{MCl}$}

Inclusion criteria (based on Petersen et al., 2001a):

1) presence of memory complaints (preferably corroborated by an informant).
2) impaired memory function documented by scores 1 standard deviation (SD) below the normal for age and education, on delayed recall of the two stories from the Logical Memory (LMd) subtest of the Wechsler Memory Scales (WMS; Garcia, 1984; Wechsler, 1969). Although in other studies performance is considered abnormal when scores are 1.5 SD below the mean for age and education matched control subjects, there is no standard cut-off to implement the memory impairment criteria (Palmer, Fratiglioni, \& Winblad, 2003; Winblad et al., 2004). A cut-off value of 1 SD was adopted considering that the use of the cut-off value of 1.5 SD could exclude subjects that from a clinical point of view suffered from MCI.

3) maintained activities of daily living; the patient should both keep the professional, social and familial activities by clinical judgement, and have no or only mild impairment in the Instrumental Activities of Daily Living (IADL) scale (Botelho, 2000; Lawton \& Brody, 1969). That is to say, no more than one item from the IADL scale suffered any changes (Pantoni et al., 2005; see below).

4) preserved general cognitive function, according to both the clinician's impression and Mini Mental State Examination (MMSE) score above cut-off (see below).

\section{Exclusion criteria:}

1) the presence of dementia, according to the DSM-IV criteria (American Psychiatric Association, 1994).

2) clinical history, imaging or laboratory tests indicating other neurological disorder; specifically, patients with history of stroke or transient ischaemic attack, brain images showing cortical or cortico-subcortical large vessel infarcts, brain hemorrhage or extensive age related white matter changes (rated $\geq 3$, in the ARWMC scale, Whalund et al., 2001); presence of psychiatric disorders, namely patients with diagnosis of major depression according to the DSM-IV criteria (American Psychiatric Association, 1994); subjects with any condition with possible impact on cognition, like systemic disease, alcohol or drug abuse were also excluded.

\section{AD}

Diagnosis of probable $\mathrm{AD}$ was based on the NINCDS-ADRDA criteria (McKhann et al., 1984). 


\section{Control group}

Inclusion criteria:

1) age $\geq 45$ years.

2) MMSE and LMd scores above cut-off (see below).

3) community dwelling, with maintained activities of daily living as evaluated by normal IADL scale, that is to say, no item from the IADL scale suffered any single change (see below).

4) no evidence for cognitive deterioration or cognitive complaints.

5) no neurological or psychiatric condition able to interfere with cognition.

Subjects with any systemic disease and/or taking psychoactive medications with possible impact on cognition as well as chronic alcohol or drug abuse did not qualify as controls.

\section{Procedures}

MCI and AD cases were subjected to clinical history, neurological examination, laboratorial evaluation and brain imaging (CT scan or NMR scan) (Knopman et al., 2001). The participating institutions use the same detailed clinical record protocol.

Patients and controls all completed the MMSE (Folstein, Folstein, \& McHugh, 1975). The normative cut-off values for the Portuguese population adjusted to education were used (Guerreiro et al., 1994). Subjects had to score above 22 if they had $\leq 11$ years of education, or above 27 if they had $>11$ years of education. These cut-off values adjusted to the education levels were similar to those found in other studies (see, for instance, Ostrosky-Solis, López-Arango, \& Ardila, 2000 and Uhlmann \& Larson, 1991, for the effect of education on MMSE cut-off scores).

IADL was applied to all patients and controls. The IADL score reflects the number of impaired activities and ranged from 0 - no impairment to 8changes in all of the items. Items were classified as not applicable if the activity had never been done before or if the subject stopped doing it for reasons other than cognitive difficulty (Tabert et al., 2002). Activities of daily living were considered preserved if no item from the IADL scale suffered any change, or mildly affected if only one item from the IADL scale was altered (Pantoni et al., 2005).

Both MCI and AD subjects had a detailed neuropsychological assessment using the Battery of Lisbon for the Assessment of Dementia (BLAD;
Garcia, 1984). The BLAD includes tests for the following cognitive domains: attention, semantic fluency, motor and graphomotor initiatives, object naming, verbal comprehension, orientation, verbal and non-verbal abstraction, visuoconstructional abilities, calculation, short and long term memory and learning.

The Blessed Dementia Scale (Blessed, Tomlinson, \& Roth, 1968; Garcia, 1984) was applied to informants of both $\mathrm{AD}$ and MCI patients, and scores of the first part (A- activities of daily living) were obtained.

The portuguese adaptation of the original 16item CVLT (Baeta, 2002; Delis et al., 1987) was administered to all subjects following standardized instructions. It starts with the oral presentation of a 16-item shopping list (List A) over five trials. Next, a second 16-item shopping list (List B) is presented once. Short-delay free recall is followed by a cued recall trial where the categories names are presented in order to facilitate recall. After a 20min interval, long-delay free and cued recall are assessed. The test finishes with a recognition trial. Indices obtained, from the original version, were: List A trial 1, List A trial 5, List A total 1-5, List B, Short-delay free recall, Short-delay cued recall, Long-delay free recall, Long-delay cued recall, Recognition hits, False Positives, Discriminability ([1-((false positives + misses $\left.) / 44)]^{*} 100\right)$, Free-recall intrusions, Cued-recall intrusions, short-delay free recall versus List A trial 5 [(SDFR-A5)/A5*100] (rapid forgetting and retroactive interference, called here Interference) and forgetting. The contrast measure used to evaluate forgetting was longdelay free recall versus List A trial 5 [(LDFR-A5)/ A5*100] (Delis et al., 1991; Lange et al., 2002; Stout et al., 1999). Semantic clustering was examined employing the method used in the CVLT-II edition (Delis, Kaplan, Kramer, \& Ober, 2000). With this method, semantic clustering measured is corrected for chance clustering using a list-based semantic clustering index. A semantic cluster is defined when two words from the same category are recalled consecutively (Bousfield, 1953). The final result is the difference between the observed clusters and the expected clusters (Observed Clusters-Expected). A positive semantic cluster index means that the subject produced more semantic clusters than expected by chance, most probably as a consequence of using a semantic clustering strategy. This correction (the Expected value) uses the target CVLT-A list as a baseline to calculate chance expected values and therefore is not influenced by the number of words recalled (Stricker, Brown, Wixted, Baldo, \& Delis, 2002). Three semantic clustering indices were calculated: Total 
learning (average clustering for the five trials A1A5), Short-delay (calculated for the short-delay free recall trial) and the Long-delay (calculated for the long-delay free recall trial). Semantic cueing has two potential effects, which were examined in the present study. One is to facilitate retrieval, enhancing the number of words recalled. The other effect of semantic cueing is to promote semantic clustering in subsequent recall.

\section{Statistical analysis}

Statistical analyses were performed with SPSS for Windows (SPSS 12; SPSS Inc, Chicago, Ill). $P$ values $<0.05$ were considered statistically significant. Demographic and neuropsychological data from the three diagnostic groups (MCI, AD and control) were analysed using the $\chi^{2}$ for categorical data, ANOVA followed by the Tukey post hoc test for numerical data with normal distribution and similar variances, or Kruskal-Wallis followed by the Dunn post hoc test for numerical data when normal distribution could not be assumed. Comparisons of neuropsychological data involving two groups were done using Student $t$ test for parametric analysis and Mann-Whitney test for nonparametric analysis. To compare learning across the five trials, and forgetting through List A trial 5, Short-delay cued recall and Long-delay cued recall, ANOVA for repeated measures was used in the 3 diagnostic groups. Comparison of the semantic cueing effects simultaneously on two dependent variables, Short-delay recall and Long-delay recall, in MCI and control subjects, was done with MANOVA. Since the clustering indices variable did not follow normal distribution, comparison of these indices in short-delay recall and long-delay recall, in MCI and control subjects, was performed with nonparametric two-way ANOVA (Maroco, 2003). Bonferroni correction for multiple comparisons was used where appropriate. Learning curves were obtained for the 3 diagnostic groups with the Prism software (Prism 4 for Windows, GraphPad software, Inc). Goodness of fitting as well as the means and 95\% confidence intervals (CIs) for the polynomial coefficients were determined. The coefficients were found statistically significantly different if they had mutually exclusive $95 \%$ CIs.

\section{RESULTS}

Demographic variables, LMd, MMSE, IADL and BDS-A scores are shown in Table 1 . No statistically significant differences among the 3 groups, controls, MCI and AD subjects, were found in education (ANOVA, $F=2.1, p>.05$ ) nor in gender distribution $\left(\chi^{2}=2.1, p>.05\right)$. Patients with AD were older than control subjects (ANOVA, $F=4.6, p<.001$; Tukey post hoc test, $p<.05$ ), but there were no age differences between the MCI patients and the other two groups. Patients with MCI had LMd, MMSE and IADL scores that were intermediate, significantly lower than the scores of controls and significantly higher than the scores of AD patients (LMd, $F=220.7, p<.001$; MMSE, $F=142.1, p<.001$; IADL, $F=98.9, p<$ .001 ; all post hoc Tukey test comparisons significant, $p<.05$; Table 1). BDS-A scores were significantly lower in MCI subjects as compared to AD $(t=-3.76, p<.01)$. No BDS-A values for controls are provided as this scale must be completed with an informant.

TABLE 1

Demographic and baseline characteristics

\begin{tabular}{|c|c|c|c|c|c|}
\hline & Control $\mathrm{n}=92$ & $M C I \mathrm{n}=70$ & $A D \mathrm{n}=21$ & Significance & Post hoc test \\
\hline Age (yrs) & $65.1 \pm 8.7(45-87)$ & $66.8 \pm 8.6(46-84)$ & $71.8 \pm 10.6(52-87)$ & $p<.05^{+}$ & $\mathrm{C}<\mathrm{AD}^{*}$ \\
\hline $\operatorname{Sex}(\mathrm{F} / \mathrm{M})$ & $54 / 38$ & $49 / 21$ & $14 / 7$ & $\mathrm{~ns}^{\widehat{े}}$ & \\
\hline Education (yrs) & $8.8 \pm 4.2(2-17)$ & $8.2 \pm 4.5(2-17)$ & $6.75 \pm 4.3(1-16)$ & $\mathrm{ns}^{+}$ & \\
\hline LMd & $12.2 \pm 2.9(6-19)$ & $4.3 \pm 2.4(0-11)$ & $0.7 \pm 0.9(0-2.5)$ & $p<.001^{+}$ & $\mathrm{C}>\mathrm{MCI}>\mathrm{AD}^{*}$ \\
\hline MMSE & $29.1 \pm 1.1(26-30)$ & $26.7 \pm 1.9(22-30)$ & $21.2 \pm 2.9(17-26)$ & $p<.001^{+}$ & $\mathrm{C}>\mathrm{MCI}>\mathrm{AD}^{*}$ \\
\hline IADL & $0 \pm 0$ & $0.6 \pm 0.8(0-1)$ & $4.3 \pm 1.7(1-6)$ & $p<.001^{+}$ & $\mathrm{C}<\mathrm{MCI}<\mathrm{AD}^{*}$ \\
\hline BDS-A & na & $1.5 \pm 0.8(0-2.5)$ & $3.2 \pm 1.7(1-7)$ & $p<.01^{*}$ & $\mathrm{MCI}<\mathrm{AD}$ \\
\hline
\end{tabular}

Note. Values are mean \pm SD (range), except Sex; C, control; MCI, Mild Cognitive Impairment; AD, Alzheimer's Disease; na, not applicable; LMd, Logical Memory delayed paragraph recall; MMSE, Mini Mental State Examination; IADL, Lawton Instrumental Activities of Daily Living; BDS-A, Blessed Dementia Scale (Part A).

${ }^{+}$ANOVA.

$\diamond \chi^{2}$.

$\mathrm{t}_{t \text {-test. }}$

*Post-hoc comparisons, Tukey test, $p<.05$ 


\section{CVLT measures}

Significant group differences were found in most CVLT measures. Patients with MCI had scores in CVLT measures that were generally lower than the scores of controls, and higher than the scores of AD patients (Table 2; see below).

\section{Learning}

The finding that the number of words correctly recalled in List A trial 1, trial 5, and the total recall trial 1-5 were significantly lower in MCI patients as compared to controls (Table 2) clearly reflects learning difficulties in MCI patients. In the last learning trial (trial A5) patients with MCI could only produce $9.6 \pm 2.7$ words, compared to $13.3 \pm 1.9$ in controls and $4.3 \pm 1.6$ in AD patients (ANOVA, $F=44.8, p<.001 ; \mathrm{C}>\mathrm{MCI}$ $>$ AD, Tukey post hoc test, $p<.05$; Table 2).

Learning across the 5 trials, A1 through A5, was compared among the 3 groups using ANOVA for repeated measures. Since the AD group was significantly older, age was taken as a covariate. As expected, there was a significant trial effect (within subject factor, Pillai's trace $=0.153, p<.0001 ; F=$ $10.7, p<.001)$ reflecting the increase in number of words correctly recalled from trial 1 to trial 5 . The interaction between age and trial was not significant (Pillai's trace $=0.042, p=.113 ; F=1.9, p=$ .161). A significant group and trial interaction effect was found (Pillai's trace $=0.340, p<.0001$; $F=14.6, p<.001$ ), meaning that the learning process was influenced by the diagnostic group. The post hoc analysis $(p<.05$, with Bonferroni CI adjustment) showed learning was significantly different among control, MCI and AD subjects.

To detail the learning difficulties we drew learning curves. In the CVLT manual, the rate of learning across the five trials is calculated as the slope of a simple linear function (Delis et al., 1987). However, and in accordance with previous studies (Warschausky, Kay, Chi, \& Donders, 2005), we found that our data were best fitted by a quadratic model $\mathrm{Y}=\mathrm{A}+\mathrm{Bx}+\mathrm{Cx}^{2}\left(r^{2}>.98\right.$ for the 3 learning curves of controls, MCI and AD subjects, Figure 1). In this equation, the coefficient $B$ represents the rate of acquisition and the coefficient $\mathrm{C}$ the rate of deceleration of learning (Warschausky et al., 2005). The intercept component was not considered in the analysis as it represents the number of words recalled in an nonexistent trial zero. Curve estimated parameters showed differences among the 3 groups for the B coefficient (mutually exclusive $95 \%$ CIs), with control subjects learning faster than MCI patients and these faster than AD patients (Table 3 ). For the $\mathrm{C}$ coefficient significant differences were found between controls and the 2 patient groups (Table 3). The greater deceleration of learning seen in the controls suggests that this group reaches a maximum list learning capacity faster than the patients groups.

TABLE 2

California Verbal Learning test scores and indices

\begin{tabular}{|c|c|c|c|c|c|}
\hline CVLT measures & Control $\mathrm{n}=92$ & $M C I \mathrm{n}=70$ & $A D \mathrm{n}=21$ & significance & Post-hoc test \\
\hline List A trial 1 & $6.9 \pm 1.72$ & $4.9 \pm 2.1$ & $2.6 \pm 1.9$ & $p<.001^{+}$ & $\mathrm{C}>\mathrm{MCI}>\mathrm{AD}^{*}$ \\
\hline List A trial 5 & $13.3 \pm 1.9$ & $9.6 \pm 2.7$ & $4.3 \pm 1.6$ & $p<.001^{+}$ & $\mathrm{C}>\mathrm{MCI}>\mathrm{AD}^{*}$ \\
\hline List A total $1-5$ & $55.1 \pm 7.8$ & $38 \pm 10.4$ & $19.6 \pm 8.3$ & $p<.001^{+}$ & $\mathrm{C}>\mathrm{MCI}>\mathrm{AD}^{*}$ \\
\hline List B & $6.5 \pm 2.1$ & $4.5 \pm 2.1$ & $2.7 \pm 1.8$ & $p<.001^{+}$ & $\mathrm{C}>\mathrm{MCI}>\mathrm{AD}^{*}$ \\
\hline Short-delay free recall & $11.2 \pm 2.7$ & $5.7 \pm 3.7$ & $1.1 \pm 1.6$ & $p<.001^{+}$ & $\mathrm{C}>\mathrm{MCI}>\mathrm{AD}^{*}$ \\
\hline Short-delay cued recall & $12.5 \pm 2.2$ & $8.1 \pm 3.1$ & $3.5 \pm 1.8$ & $p<.001^{+}$ & $\mathrm{C}>\mathrm{MCI}>\mathrm{AD}^{*}$ \\
\hline Long-delay free recall & $11.8 \pm 2.4$ & $6.1 \pm 4.1$ & $0.6 \pm 1.3$ & $p<.001^{+}$ & $\mathrm{C}>\mathrm{MCI}>\mathrm{AD}^{*}$ \\
\hline Long-delay cued recall & $12.7 \pm 2.2$ & $7.7 \pm 3.2$ & $3.3 \pm 2.0$ & $p<.001^{+}$ & $\mathrm{C}>\mathrm{MCI}>\mathrm{AD}^{*}$ \\
\hline Recognition hits & $14.8 \pm 1.4$ & $13.2 \pm 2.8$ & $11.2 \pm 3.4$ & $p<.001^{+}$ & $\mathrm{C}>\mathrm{MCI}>\mathrm{AD}^{*}$ \\
\hline False Positives & $0.8 \pm 1.2$ & $5.6 \pm 4.9$ & $10.4 \pm 5.2$ & $p<.001^{+}$ & $\mathrm{C}<\mathrm{MCI}<\mathrm{AD}^{*}$ \\
\hline Free recall intrusions & $2.6 \pm 3.1$ & $7.0 \pm 8.0$ & $9.7 \pm 11.0$ & $p<.001^{\diamond}$ & $\mathrm{C}<\mathrm{MCI}, \mathrm{AD}^{\star}$ \\
\hline Cued recall intrusions & $1.1 \pm 1.7$ & $5.7 \pm 4.6$ & $10.5 \pm 6.5$ & $p<.001^{\diamond}$ & $\mathrm{C}<\mathrm{MCI}, \mathrm{AD}^{\star}$ \\
\hline Discriminability $(\%)\left[1-((\mathrm{FP}+\mathrm{M}) / 44]^{*} 100\right.$ & $95.6 \pm 4.3$ & $81.2 \pm 12.0$ & $65.6 \pm 9.3$ & $p<.001^{\diamond}$ & $\mathrm{C}>\mathrm{MCI}>\mathrm{AD}^{\star}$ \\
\hline Interference $(\%)((\mathrm{SDFR}-\mathrm{A} 5) / \mathrm{A} 5)^{*} 100$ & $15.3 \pm 16.0$ & $44.2 \pm 27.0$ & $75.4 \pm 37.8$ & $p<.001^{\widehat{ }}$ & $\mathrm{C}<\mathrm{MCI}<\mathrm{AD}^{\star}$ \\
\hline Forgetting $(\%)(($ LDFR-A5)/A5)*100 & $10.7 \pm 14.7$ & $42.6 \pm 32.7$ & $87.5 \pm 32.2$ & $p<.001^{\diamond}$ & $\mathrm{C}<\mathrm{MCI}<\mathrm{AD}^{\star}$ \\
\hline
\end{tabular}

Note. Values are mean \pm SD. C, control; MCI, Mild Cognitive Impairment; AD, Alzheimer's Disease; FP, false positives; M, misses; SDFR, short-delay free recall; LDFR, long-delay free recall; A5, learning in Trial 5.

${ }^{+}$ANOVA.

${ }^{\wedge}$ Kruskal-Wallis test.

*Post-hoc comparisons, Tukey test for parametric data $p<.05$.

${ }^{\star}$ Post-hoc comparisons, Dunn test for nonparametric data, $p<.05$. 


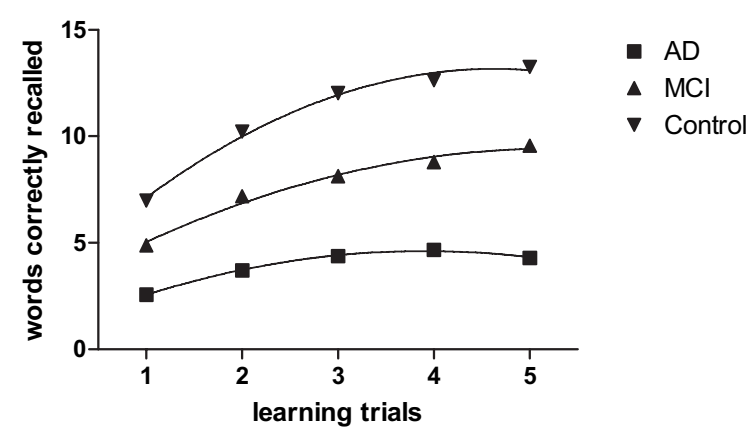

Figure 1. Learning curves fitted to CVLT learning trials A1 through A5, in MCI, controls and AD patients. Learning curves were significantly different for the 3 diagnostic groups (see text).

TABLE 3

Curve fitting parameters of the learning curves

\begin{tabular}{llll}
$\begin{array}{l}\text { Curve } \\
\text { parameters } \\
\text { estimated }\end{array}$ & \multicolumn{1}{c}{$\begin{array}{c}\text { Control } \\
\mathrm{n}=92\end{array}$} & \multicolumn{1}{c}{$\begin{array}{c}\text { MCI } \\
\mathrm{n}=70\end{array}$} & \multicolumn{1}{c}{$\begin{array}{c}A D \\
\mathrm{n}=21\end{array}$} \\
\hline $\mathrm{B}$ & 4.25 & 2.54 & 1.91 \\
& {$[4.18-4.32]$} & {$[2.46-2.62]$} & {$[1.88-1.93]$} \\
$\mathrm{C}$ & -0.46 & -0.24 & -0.24 \\
& {$[(-0.47)-(-0.45)]$} & {$[(-0.25)-(-0.23)][(-0.25)-(-0.24)]$} \\
\hline
\end{tabular}

Note. Values are mean [95\% confidence interval]. Coefficient B was significantly different among the 3 groups, with control subjects learning faster than MCI patients and these faster than $\mathrm{AD}$ patients. For the coefficient $\mathrm{C}$, significant differences were found between control and the 2 patient groups, with greater deceleration of learning in the controls.

\section{Semantic clustering}

Patients with MCI might have learning difficulties due to inefficient use of learning strategies. Semantic clustering represents the degree to which subjects apply an active strategy of reorganizing words through categorical groups rather than recalling them in another order. Semantic cueing reveals the semantic structure of the list facilitating retrieval especially in cases when free recall is difficult. Semantic clustering indices were calculated in learning (Total learning) and recall trials (Shortdelay and Long-delay). Since the semantic clustering index gives little information when few items are recalled, and $\mathrm{AD}$ patients could recall very few words from the list ( $40 \%$ obtained scores $\leq 3$ on List A trial 5; $55 \%$ scored 0 on short-delay free recall), this group was excluded from subsequent analysis. MCI patients had lower semantic clustering indices than the control group. Significant differences were found on the Total learning $(\mathrm{MCI}=0.5 \pm 1.0$, $\mathrm{C}=2.0 \pm 1.8$; Mann-Whitney test, $U=1243, p<$ $.001)$, on the Short-delay recall $(\mathrm{MCI}=0.9 \pm 1.3$, $\mathrm{C}=3.5 \pm 2.6 ; U=1099, p<.001)$ and on the Long- delay recall $(\mathrm{MCI}=1.3 \pm 1.8, \mathrm{C}=4.1 \pm 2.6 ; U=$ $1044, p<.001)$, reflecting a feeble use of the semantic structure of the list in MCI patients (Figure 2). To study the effect of semantic cueing on subsequent semantic clustering, the semantic clustering indices on short-delay recall and long-delay recall were compared in control and MCI subjects. The semantic clustering indices increased from short delay recall to long-delay recall both in controls and in MCI patients (Figure 2). The diagnosis influenced the short and long delay semantic clustering indices (non parametric two-way ANOVA, $\mathrm{H}=78.49, p<.001)$, which were lower in MCI patients than in controls. Furthermore, the semantic clustering indices were higher on long-delay recall than on the short-delay recall $(\mathrm{H}=2.39, p<$ $.0001)$, reflecting an improvement in semantic cluster organization along the test. However, no significant interaction between the diagnosis and the delay condition was found $(\mathrm{H}=0.07, p>.05)$, meaning that the improvement in semantic clustering from short-delay to long-delay was not significantly different in MCI patients as compared to controls.

We next studied the effect of semantic cueing on the success of list recall in MCI patients and controls. Analysis of the effect of the diagnosis, MCI patients or controls, and the effect of the recall condition, free or cued, on the number of words recalled in both short-delay and long-delay trials was performed with MANOVA. The diagnosis influenced the number of words recalled (MANOVA, Pillai's trace $=0.91, p<.0001$ ), either in short-delay trial $(F=224.58, p<.0001)$ or in longdelay trial $(F=253.84, p<.0001)$, the success of recall being lower in MCI patients than controls. The recall condition also influenced the number of words recalled (Pillai's trace $=0.09, p<.0001$ ), either in short-delay trial $(F=31.67, p<.0001)$ or

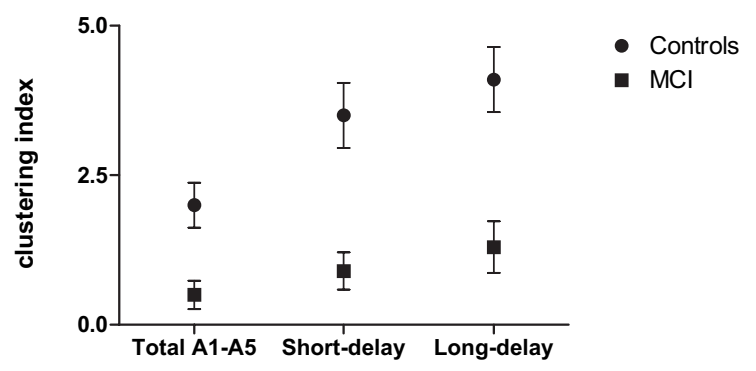

Figure 2. Semantic clustering indices from CVLT total A1-A5 trials, short-delay free recall, and long-delay free recall, in MCI patients and controls. The means and 95\% confidence intervals are depicted. All semantic clustering indices were lower in MCI patients than in controls, but the improvement in semantic clustering (namely from short delay to long-delay recall) was not significantly different in MCI patients and controls (see text). 
in long-delay trial $(F=15.86, p<.0001)$, more words being recalled in the cued condition in both short and long delay. However, no significant interaction between the diagnosis and recall condition was found (Pillai's trace $=0.01, p=.225$ ), either in the short-delay trial $(F=2.9, p=.9)$ or in the long-delay trial $(F=1.6, p=.2)$, meaning that MCI patients did not benefit from cueing differently than the controls.

\section{Rate of forgetting}

The Long-delay free recall score was substantially lower in patients with MCI, $6.1 \pm 4.1$ words, as compared with controls, $11.8 \pm 2.4$ words, whereas patients with AD could only spontaneously remember $0.6 \pm 1.3$ words after long delay, $78 \%$ of AD patients scoring zero in this trial (ANOVA, $F=134.1, p<.001 ; \mathrm{C}>\mathrm{MCI}>\mathrm{AD}$, Tukey post hoc test, $p<.05$; Table 2). Differences were also significant in Long-delay cued recall with MCI patients recalling $7.7 \pm 3.2$ and the control group recalling $12.7 \pm 2.2$ words and $\mathrm{AD}$ patients $3.3 \pm$ 2.0 (ANOVA, $F=134.2, p<.001 ; \mathrm{C}>\mathrm{MCI}>\mathrm{AD}$, Tukey post hoc test, $p<.05$; Table 2 ). The poor score obtained by MCI patients in long-delay recall was not only attributable to poor achievement during the learning phase of the test. The forgetting index [(LDFR-A5)/A5*100], which relates the long-delay free recall score with the List A trial 5 score, was higher in MCI patients, $42.6 \pm 32.7 \%$, as compared with controls, $10.7 \pm 14.7 \%$, and very high in AD patients, $87.5 \pm 32.2 \%$ (Kruskal-Wallis $\mathrm{H}=54.33, p<.001 ; \mathrm{C}<\mathrm{MCI}<\mathrm{AD}$, Dunn's post hoc test, $p<.05$; Table 2). To detail the forgetting pattern, ANOVA for repeated measures was conducted comparing the number of words recalled in List A trial 5, Short-delay cued recall and Longdelay cued recall among the 3 groups. As expected, there was a significant within subject factor effect (Pillai's trace $=0.16, p<.0001 ; F=23.5, p<.001$ ) reflecting the forgetting process. A significant diagnostic group and trial interaction effect was found (Pillai's trace $=0.09, p<.0001 ; F=5.0, p<.001$ ), meaning that the forgetting process was significantly influenced by the diagnostic group, and post hoc analysis showed that forgetting was significantly different among control, MCI and AD subjects (Tukey test, $p<.05$ ).

\section{Recall errors}

Patients with MCI were more vulnerable to recall intrusions than the controls, both under free (Kruskall-Wallis $\mathrm{H}=28.5, p<.001$; $\mathrm{C}<\mathrm{MCI}, \mathrm{AD}$,
Dunn post hoc test, $p<.001$; Table 2) and cued recall conditions (Kruskall-Wallis $\mathrm{H}=70.7 p<$ .001; C < MCI,AD, Dunn post hoc test, $p<.001$; Table 2).

\section{Recognition}

Recognition hits were significantly different in the three groups with the control group scoring near ceiling in this task (ANOVA, $F=22.4, p<.001$; C $>$ MCI $>$ AD, Tukey post hoc test, $p<.05$; Table 2). The number of false positives was larger in MCI patients, $5.6 \pm 4.9$, than in controls, $0.8 \pm 1.2$, and very high in AD patients, $10.4 \pm 5.2$ (ANOVA, $F=$ 74.8, $p<.001 ; \mathrm{C}<\mathrm{MCI}<\mathrm{AD}$, Tukey post hoc test, $p<.05$; Table 2).

The discriminability index indicates the ability to recognize the true items and reject the items that do not belong to the list. The discriminability index was highest in controls, $95.6 \pm 4.3 \%$, intermediate in MCI subjects, $81.2 \pm 12.0$, and lowest in AD patients, $65.6 \pm 9.3 \%$ (Kruskall-Wallis $\mathrm{H}=104.9$, $p<.001 ; \mathrm{C}>\mathrm{MCI}>\mathrm{AD}$, Dunn post hoc test, $p<$ .05 ; Table 2).

\section{DISCUSSION}

The currently used diagnostic criteria for MCI emphasize the presence of consolidation deficits, typically requiring a low score on the delayed recall task of a prose passage (Petersen et al., 2001b). The present study shows that in addition to enhanced forgetting, subjects with MCI have prominent learning difficulties and deficient use of semantic clustering strategies that may contribute to their memory troubles.

To assess in detail verbal learning and memory, we used an established test, the CVLT, which provides indices concerning rate of learning, learning strategies, recall, recall errors and recognition (Delis et al., 1987). In particular, the use of a semantic structured list enables the study of semantic strategies in learning and recall. In the present study, the CVLT was applied to MCI patients, and compared with normal control subjects, as well as with AD patients.

\section{Acquisition}

Patients with MCI had lower learning performances on trials A1-A5 than controls, but higher than AD patients. Detailed analysis of the learning curves confirmed that MCI subjects had a slower rate of acquisition than the control 
group, and faster than AD patients. Differences in the late phase of the learning curve were also detected among the groups; AD and MCI patients had lower decelerating rates than the controls, being unable to reach in the five trials the maximum capacity for list learning, most likely because of poorer learning resources. Differences between MCI and AD concerning acquisition capacity have been found in some studies but not in others. Crowell, Luis, Vanderploeg, Schinka, \& Mullan (2002) detected no differences among MCI subjects, AD patients and controls in the rate of acquisition on the CERAD list learning task, despite the differences in the number of words recalled on each trial. Failure to see any significant differences in this study can be related to the characteristics of the list learning task (ten unrelated words and three learning trials) or to the small number of subjects evaluated. Others (Estévez-González, Kulisevsky, Boltes, Otermín, \& García- Sánchez, 2003), using the Rey's Auditory Verbal Learning Test (RAVLT), showed significant differences in the learning curves between MCI patients, AD patients and controls, as observed in the present study.

\section{Semantic clustering}

Taking advantage of the semantic structure of the material to learn, when this structure does exist, is an automatic behavior in healthy subjects (Bousfield, 1953). The use of the semantic structure of the list during the learning process is revealed by analysis of the semantic clusters. Semantic clustering reflects conceptual organization and is known to have a positive effect on encoding and retrieval success (Delis, Kaplan \& Kramar, 1988). In the present study, although MCI patients could use some clustering (the positive mean value of the semantic clustering index means that the clustering is above chance), they employed less semantic clustering strategies than the control group.

The researcher may provide the subject with the semantic structure of the list, by performing a categorical cued recall trial. It is thus possible to study the effect of external semantic cueing both on semantic clustering and on the number of words recalled in subsequent trials. The CVLT includes a cued recall task after the initial free recall and before the delayed free recall. This cued recall is known to increase semantic clustering in the delayed recall task in healthy participants (Shear, Wells, \& Brock, 2000). We confirmed this improvement in semantic clustering from short-delay to long-delay recall, however, it was not significantly different in MCI patients as compared to controls. This means that patients with MCI could indeed benefit from the cued recall to boost subsequent semantic clustering. Semantic cueing also facilitated the number of words recalled, both in the short-delay trial and in the long-delay trial. Again, MCI patients benefited from semantic cueing to increase the number of words subsequently recalled, in a way not statistically different from the controls.

The reasons for the poor spontaneous use of semantic strategies in MCI patients are not clear. It is not possible, based solely on clustering strategies, to distinguish between encoding and recall deficits since semantic clusters are assessed on free recall (Vanderploeg, Crowell, \& Curtiss, 2001). Deficiency of the semantic strategy may reflect impaired executive functioning, difficulties in detecting the semantic structure of the list, that is to say, a semantic memory deficit (Howieson \& Lezak, 1995), or both. If MCI patients do not use the semantic structure of the list, partly because of a strategy implementation deficit, then if the semantic structure is made explicit with cueing, it should be used more easily afterwards (see Knoke, Taylor, \& Saint-Cyr, 1998, for the effect of cueing in Parkinson's disease). This was the pattern found here, suggesting an unsatisfactory use of executive resources. Executive deficits have already been described in MCI patients (Crowell et al., 2002), and were considered useful in predicting those who will progress to AD (Chen et al., 2000). Semantic memory deficits may also be present in MCI patients and probably contribute to poor semantic categorization (Dudas et al., 2005). Certainly multiple brain structures are activated in declarative memory operations, either encoding or retrieval, particularly the left prefrontal neocortex, temporal neocortex, hippocampus and anterior cingulated areas, as well as precuneus, thalamus and cerebellum (see review by Nyberg \& Cabeza, 2000) and these brain structures may be affected in patients with MCI. For instance, neurochemical changes relevant for memory processes, namely upregulation of choline acetyltransferase activity, were reported in the frontal cortex as well as medial temporal lobe structures (DeKosky et al., 2002). In accordance with the notion of a widespread pathological process, if detailed neuropsychological testing is performed, MCI patients frequently have mild deficits in cognitive domains beyond memory, namely semantic fluency, complex language abilities, calculation and motor initiative (Ribeiro, Guerreiro, \& de Mendonça, 2006). 


\section{Forgetting}

As expected from the inclusion criteria, the rate of forgetting was higher in MCI than in controls. Prominent forgetting is the hallmark of $\mathrm{AD}$, and in this sample of mildly demented cases (MMSE = $21.2 \pm 2.9$ ), as much as $80 \%$ of the $\mathrm{AD}$ patients scored zero in long delay free recall. As previously described, even the shorter 9-item CVLT version is a very challenging test to AD patients (Fox, Olin, Erblich, Ippen, \& Schneider, 1998).

\section{Recognition}

When performance in recognition tasks is clearly better than in free recall, this is generally taken as a mark of retrieval impairment (Lezak, 1995). In MCI patients the mean number of recognition hits $(13.2 \pm 2.8)$ was higher than the mean number of correct words in delayed free recall $(6.1 \pm 4.1)$. However, this does not mean a good recognition performance, because the number of false positive answers in recognition was also high (5.6 \pm 4.9$)$. Altogether, the number of recognition hits was lower and the number of false positive answers was higher in MCI subjects as compared to controls, and consequently the discriminability index (the net ability to recognize the true items and to reject the items that do not belong to the list), was disturbed in MCI patients.

\section{Recall errors}

False positive errors were not the only type of recall errors MCI subjects seem vulnerable to; the number of intrusion errors both in free and cued recall was significantly higher than in controls and similar to the AD group.

Altogether, MCI patients scored lower than controls, and higher than $\mathrm{AD}$ patients, on almost every CVLT measure. A recent study (Perri et al., 2005), with another verbal learning task, also revealed MCI deficits in list learning and ineffective use of the list semantic structure. Because there were no cued recall trials in the abovementioned study, it is impossible to know if the MCI patients could also have taken advantage of semantic cueing.

Certainly, the present study is not designed to address whether MCI subjects do correspond to the initial phase of $\mathrm{AD}$. But the observation that California Verbal Learning test scores and indices in MCI patients were generally intermediate between the values in controls and in patients with $\mathrm{AD}$ is consistent with the notion that amnestic MCI (as defined by the type of criteria used in the present study), may correspond to a very initial phase of AD (Morris, \& Cummings, 2005; Petersen $\&$ Bennett, 2005). In fact it was recently reported that as much as $62 \%$ of patients with MCI met pathological criteria for intermediate or high likelihood of AD at the time of death (Bennett, Schneider, Bienias, Evans, \& Wilson, 2005).

There are other limitations of the present study. Because only a verbal learning test was used, the generalizability of the learning deficits to nonverbal processes is uncertain. Also the control group had a shorter neuropsychological assessment than the patients, precluding the study of possible correlations between learning and memory impairments in the CVLT and performance in other cognitive domains.

The CVLT proved a very sensitive test to detect memory deficits in MCI. This study confirmed that MCI patients suffer from severe consolidation difficulties but also revealed less studied aspects of memory dysfunction, like inadequate acquisition and a feeble use of semantic strategies. Most importantly, it suggests that MCI patients retain the ability to improve learning, if helped by the use of appropriate semantic cueing interventions.

Original manuscript received 18 August 2005 Revised manuscript accepted 24 January 2006 First published online 20 October 2006

\section{REFERENCES}

American Psychiatric Association (1994). Diagnostic and Statistical Manual of Mental Disorders (4th ed.). Washington DC: American Psychiatric Association.

Arnáiz, E., \& Almkvist, O. (2003). Neuropsychological features of mild cognitive impairment and preclinical Alzheimer's disease. Acta Neurologica Scandinavica, 107, 34-41.

Baeta, E. (2002). Bateria para avaliação neuropsicológica de adultos com epilepsia. Psicologia, 16, 79-96.

Bennett, D. A., Schneider, J. A., Bienias, J. L., Evans, D. A., \& Wilson, R.S. (2005). Mild cognitive impairment is related to Alzheimer disease pathology and cerebral infarctions. Neurology, 64, 834-841.

Blessed, G., Tomlinson, B. E., \& Roth, M. (1968). The association between quantitative measures of dementia and of senile change in the cerebral grey matter of elderly subjects. The British Journal of Psychiatry, 114, 797-811.

Botelho, M. A. (2000). Autonomia funcional em idosos. Porto: Laboratórios Bial.

Bousfield, W. A. (1953). The occurrence of clustering in recall of randomly arranged associates. The Journal of General Psychology, 49, 229-240.

Chen, P., Ratcliff, G., Belle, S. H., Cauley, J. A., deKosky, \& Gangulli, (2000). Cognitive tests that best discriminate between presymptomatic AD and those who remained nondemented. Neurology, 55, 1847-1853. 
Crowell, T. A., Luis, C. A., Vanderploeg, R. V., Schinka, J. A., \& Mullan M. (2002). Memory patterns and executive functioning in Mild Cognitive Impairment and Alzheimer's Disease. Aging Neuropsychology and Cognition, 9, 288-297.

de Mendonça, A., Guerreiro, M., Ribeiro, F., Mendes, T., \& Garcia C. (2004). Mild Cognitive Impairment. Focus on diagnosis. Journal of Molecular Neuroscience, 23, 13-17.

DeKosky, S. T., Ikonomovic, M. D., Styren, S. D., Beckett, L., Wisniewski, S., Bennett, D. A., Cochran, E. J., Kordower, J. H., \& Mufson, E. J. (2002). Upregulation of choline acetyltransferase activity in hippocampus and frontal cortex of elderly subjects with mild cognitive impairment. Annals of Neurology, $51,145-155$.

Delis, D. C., Kaplan, E., Kramer, J. H., \& Ober, B. A. (1988). Integrating clinical assessment with cognitive neuroscience: Construct validation of the California Verbal Learning Test. Journal of Consulting and Clinical Psychology, 56, 123-130.

Delis, D. C., Kaplan, E., Kramer, J. H., \& Ober, B. A. (2000). California Verbal Learning Test:-II. (2nd ed.). The Psychological Corporation, San Antonio, TX.

Delis, D. C., Kramer, J. H., Kaplan, E., \& Ober, B. A. (1987). California Verbal Learning Test: Research edition. New York: The Psychological Corporation.

Delis, D. C., Massman, P. J., Butters, N., Salmon, D. P., Cermak, L. S., \& Kramer, J. H. (1991). Profiles of demented and amnesic patients on the California Verbal Learning Test: Implications for the assessment of memory disorders. Psychological Assessment, 3, 19-26.

Dudas, R. B., Claghe, F., Thompson, S. A., Graham, K. S., \& Hodges, J. R. (2005). Episodic and semantic memory in mild cognitive impairment. Neuropsychologia, 43, 1266-1276.

Estévez-González, A., Kulisevsky, J., Boltes, A., Otermín, P., \& García- Sánchez, C. (2003). Rey verbal learning test is a useful tool for differential diagnosis in the preclinical phase of Alzheimer's disease: Comparison with mild cognitive impairment and normal aging. International Journal of Geriatric Psychiatry, 18, 1021-1028.

Folstein, M. F., Folstein, S. E., \& McHugh, P. R. (1975). Mini-Mental State. A practical method for grading the cognitive state of patients for the clinician. Journal of Psychiatric Research, 12, 189-98.

Fox, L. S., Olin, J. T., Erblich, J., Ippen, C. G., \& Schneider, L. S. (1998). Severity of cognitive impairment in Alzheimer's disease affects list learning using the California Verbal learning Test (CVLT). International Journal of Geriatric Psychiatry, 13, 544-549.

Garcia, C. (1984). Alzheimer's disease: Difficulties in clinical diagnosis. PhD Dissertation, Faculty of Medicine, University of Lisbon, Lisbon.

Grundman, M., Petersen, R. C., Ferris, S. H., Thomas, R. G., Aisen, P. S., Bennett, D. A., Foster, N. L., Jack, C. R. Jr, Galasko, D. R., Doody, R., Kaye, J., Sano, M., Mohs, R., Gauthier, S., Kim, H. T., Jin, S., Schultz, A. N., Schafer, K., Mulnard, R., van Dyck, C. H., Mintzer, J., Zamrini, E. Y., CahnWeiner, D., Thal, L. J.; for the Alzheimer's Disease Cooperative Study. (2004). Mild Cognitive Impairment can be distinguished from Alzheimer disease and normal aging for clinical trials. Archives of Neurology, 61, 59-66.
Guerreiro, M., Silva, A. P., Botelho, M. A., Leitão, O., Castro Caldas, A., \& Garcia, C. (1994). Adaptação à população portuguesa na tradução do "MiniMental State Examination” (MMSE). Revista Portuguesa de Neurologia. 1, 9.

Howieson, D. B., \& Lezak, M. D. (1995). Separating memory from other cognitive problems. In A.D. Baddeley, B.A. Wilson \& F.N. Watts (Eds.), Handbook of memory disorders. Chichester, UK: Wiley.

Knoke, D., Taylor, A. E., \& Saint-Cyr, J. A. (1998). The differential effects of cueing on recall in Parkinson's disease and normal subjects. Brain and Cognition, 38, 261-274.

Knopman, D. S., DeKosky, S. T., Cummings, J. L., Chui, H., Corey-Bloom, J., Relkin, N., Small, G. W., Miller, B., \& Stevens, J. C. (2001). Practice parameter: Diagnosis of dementia (an evidence-based review). Neurology, 56, 1143-1153.

Kramer, J. H., Levin, B. E., Brandt, J., \& Delis,. D. C. (1989). Differentiation of Alzheimer's, Huntington's, and Parkinson's disease patients on the basis of verbal learning characteristics. Neuropsychology, 3, 111-120.

Lange, K. L., Bonde, M. W., Salmon, D. P., Galasko, D., Dellis, D. C., Thomas, R. G, \& Thal, L. J. (2002) Decline in verbal memory during preclinical Alzheimer's disease: Examination of the effect of APOE genotype. Journal of the International Neuropsychological Society, 8, 943-455.

Lawton, M. P., \& Brody, E. M. (1969). Assessment of older people: Self-maintaining and instrumental activities of daily living. Gerontologist, 9, 179-186.

Lezak, M. D. (1995). Neuropsychological assessment (3rd ed.). New York: Oxford University Press.

Maroco J. (2003). Análise estatística. Com utilização do SPSS (2nd ed., pp. 188-195). Lisboa: Edições Sílabo.

McKhann, G., Drachman, D., Folstein, M., Katzman, R., Price, D., \& Stadlan, E. M. (1984). Clinical diagnosis of Alzheimer's disease: Report of the NINCDSADRDA Work Group. Neurology, 34, 939-944.

Morris, J. C., Cummings, J. (2005). Mild cognitive impairment (MCI) represents early-stage Alzheimer's disease. Special Report from the challenging views of Alzheimer's disease. Journal of Alzheimer's Disease, 7, 235-239

Nyberg, L. \& Cabeza, R. (2000). Brain imaging of memory. In E. Tulving \& F. I. M. Craik (Eds.), The Oxford handbook of memory (pp. 501-519). Oxford: Oxford University Press.

Ostrosky-Solis, F., López-Arango, G., \& Ardila, A. (2000). Sensitivity and specificity of the Mini-Mental state examination in a Spanish-speaking population. Applied Neuropsychology, 7, 25-31.

Palmer, K., Fratiglioni, L., \& Winblad, B. (2003). What is mild cognitive impairment? Variations in definitions and evolution of nondemented persons with cognitive impairment. Acta Neurologica Scandinavica, 179, 14-20.

Pantoni, L., Basile, A. M., Pracucci, J., Asplund, K., Bogousslavsky, J., Chabriat, H., Erkinjuntti, T., Fazekas, F., Ferro, J. M., Hennerici, M., O'Brien, J., Scheltens, P., Visser, M. C., Whalund, L. O., Waldemar, G., Wallin, A., \& Inzitari, D., on behalf of the Ladis study group. (2005). Impact of age related cerebral white matter changes on the transition to disability-The Ladis Study: Rationale, design and methodology. Neuroepidemiologia, 24, 51-62. 
Perri, R., Carlesimi, G. A., Serra, L., Caltagirone, C., and the Early Diagnosis Group of the Italian Interdisciplinary Network on Alzheimer's Disease. (2005). Characterization of memory profile in subjects with Amnestic Mild Cognitive Impairment. Journal of Clinical and Experimental Neuropsychology, 27, 1033-1055.

Petersen, R. C., \& Bennett, D. (2005). Mild Cognitive Impairment: Is it Alzheimer's disease or not? Special report from the challenging views of Alzheimer's disease. Journal of Alzheimer's Disease, 7, 241-245.

Petersen, R. C., Doody, R., Kurz, A., Mohs, R. C., Morris, J. C., Rabins, P. V., Ritchie, K., Rossor, M., Thal, L., \& Winblad, B. (2001a). Current concepts in mild cognitive impairment. Archives of Neurology, 58, 1985-1992.

Petersen, R. C., Smith, G. E., Waring, S. C., Ivnik, R. J., Tangalos, E. G., \& Kokmen, E. (1999). Mild cognitive impairment. Archives of Neurology, 56, 303-308.

Petersen, R. C., Stevens, J. C., Ganguli, M., Tangalos, E. G., Cummings, J.L., DeKosky, S.-T. (2001b). Practice parameter: Early detection of dementia: Mild cognitive impairment (an evidence-based review): Report of the Quality Standards Subcommittee of the American Academy of Neurology. Neurology, 56, 1133-42.

Ribeiro, F., Guerreiro, M., \& de Mendonça, A. (2006). Mild Cognitive Impairment: deficits in cognitive domains other than memory. Dementia and Geriatric Cognitive Disorders, 21, 284-290.

Shear, P. K., Wells, C. T., \& Brock, A. M. (2000) The effect of semantic cuing on CVLT performance in healthy participants. Journal of Clinical and Experimental Neuropsychology, 122, 649-655.

Stout, J. C., Bondi, M. W., Jernigan, T. L., Archibald, S. L., Delis, D. C. \& Salmon D. P. (1999). Regional cerebral loss associated with verbal learning and memory of the Alzheimer type. Neuropsychology, 13, 188-197.

Stricker, J. L., Brown, G. G., Wixted, J., Baldo, J. V., \& Delis, D. C. (2002). New semantic and serial clustering indices for the California Verbal Learning Test-Second Edition: Background, rationale, and formulae. Journal of the International Neuropsychological Society, 8, 425-435.

Tabert, M. H., Albert, S. M., Borukhova-Milov, L., Camacho, Y., Pelton, G., Liu, X., Stern, Y., \& Devanand, D. P. (2002). Functional deficits in patients with mild cognitive impairment: Prediction of AD. Neurology, 58, 758-764.

Uhlmann, R. F., \& Larson, E. B. (1991). Effect of education on the mini-mental state examination as a screening test for dementia. Journal of American Geriatric Society, 39, 876-880.

Vanderploeg, R. D. Crowell, T. A., \& Curtiss, G. (2001). Verbal learning and memory deficits in traumatic brain injury: Encoding, consolidation, and retrieval. Journal of Clinical and Experimental Neuropsychology, 23, 185-195.

Wahlund, L. O., Barkhof, F., Fazekas, F., Bronge, L., Augustin, M., Sjogren, M., Wallin, A., Ader, H., Leys, D., Pantoni, L., Pasquier, F., Erkinjuntti, T., Scheltens, P.; European Task Force on Age-Related White Matter Changes. (2001). A new rating scale for age-related white matter changes applicable to MRI and CT. Stroke, 32, 1318-1322.

Warschausky, S., Kay, J. B., Chi, P. L., \& Donders, J. (2005). Hierarchical linear modeling of California Verbal Learning Test-Children's Version learning curve characteristics following childhood traumatic head injury. Neuropsychology 19, 193-198.

Wechsler, D. (1969). Manuel de l'échelle clinique de mémoire. Paris. Centre de Psychologie Appliquée.

Winblad, B., Palmer, K., Kivipelto, M., Jelic, V., Fratiglioni, L., Wahlund, L.-O., Nordberg, A., Bäckman, L., Albert, M., Almkvist, O., Arai, H., Basun, H., Blennow, K., de Leon, M., DeCarli, C., Erkinjuntti, T., Giacobini, E., Graff, C., Hardy, J., Jack, C., Jorm, A., Ritchie, K., Van Duijn, C., Visser, P., \& Petersen, R.C. (2004). Mild Cognitive Impairment: Beyond controversies, towards a consensus: report of the International Working Group on Mild Cognitive Impairment. Journal of Internal Medicine, 256, 240-246. 
Copyright of Journal of Clinical \& Experimental Neuropsychology is the property of Psychology Press (UK) and its content may not be copied or emailed to multiple sites or posted to a listserv without the copyright holder's express written permission. However, users may print, download, or email articles for individual use. 